\title{
Effective Utilization of STEM Imaging Capability in FIB for Physical Failure Analysis on 20nm \& 14nm Transistor Nodes in Semiconductor Wafer Foundries
}

\author{
Wayne Zhao ${ }^{1}$, Donald Nedeau ${ }^{1}$, Satish Kodali ${ }^{1}$, Jiang Huang ${ }^{2}$, Chong Khiam $\mathrm{Oh}^{1}$, Seng-Keat Lim ${ }^{1}$, \\ Raghaw Rai ${ }^{1}$, Zhi-Hong $\mathrm{Mai}^{3}$, and Jeffrey $\mathrm{Lam}^{3}$ \\ ${ }^{1}$ Product Support Engineering, New Technology Prototyping, Technology Development, \\ GLOBALFOUNDRIES, Malta, New York, USA. \\ ${ }^{2}$ Engineering Analytical Services, Quality and Reliability Assurance, GLOBALFOUNDRIES, Malta, \\ New York, USA. \\ ${ }^{3}$ Product Analysis Engineering, New Technology Prototyping, Technology Development, \\ GLOBALFOUNDRIES, Singapore.
}

With semiconductor transistor nodes shrinking to $20 \mathrm{~nm} / 14 \mathrm{~nm}$ and beyond, physical failure analyses (PFA) requires frequent transmission electron microscopy (TEM) to discern detailed features of interests in order to pin-point root-causes conclusively for major yield detractors [1 5]. Occasionally, bottle-neck can happen, when severe tool / process excursions occur, leading to spikes in TEM needs. This overwhelming demand for TEM (even if just temporary) justified the necessity to explore alternative means of cross-sectional defect identification, especially at wafer foundries, to effectively ensure that high TEM priority is granted to those truly deserving without compromising turnaround time and business needs for other modules with lower TEM priority. Fully utilizing the scanning transmission electron microscopy (STEM) mode coupled with a focused ion beam (FIB) is one such alternative.

Fundamentally, the major contributor to the difference in the imaging quality of "fuzzy" SEM images versus "sharp" TEM images is the existence of an energy-activated zone beneath the surface in the bulk SEM sample. Such effect is minimized in TEM, as the sample is cross-sectioned into a thin lamella. Additionally, TEM/STEM with a $200 / 300 \mathrm{kV}$ beam has better resolution than that of SEM beam at $30 \mathrm{kV}$ and also better contrast, e.g., due to diffraction contrast. STEM imaging inside FIB bridges the gap to balance the limited SEM imaging quality and constraints on tool availability of TEM. Fortunately, synchronized with semiconductor wafer-fab development, more and more state-of-the-art FIBs are now usually equipped with STEM detectors. However, the STEM mode in FIB is either rarely used or primarily employed to screen TEM sample quality in the majority of PFA cases. Demonstrated here are successful examples from real case studies at a wafer foundry to prove the effectiveness of this alternative approach.

Presented in Figure-1 are high angle annular dark field (HAADF) STEM images, taken at 30kV from a FIB (Helios $450 \mathrm{ML}$ ), for a contact in a $14 \mathrm{~nm}$ SRAM device, with a relatively thick lamella in (a), and a thin lamella in (b). As a comparison, Fig-2 displayed a HAADF-STEM image in a TEM with $200 \mathrm{kV}$. Even though the resolution of STEM imaging at $30 \mathrm{kV}$ inside a FIB definitely less competitive against the STEM mode in an Analytical TEM with $200 / 300 \mathrm{kV}$, this is still very applicable where relatively large features are of interests, such as case studies for contact-open / junction-fill for back-end-of-line (BEOL) interconnects, or other testing / diagnostic macros with long / wide pads, etc. By using STEM appropriately for the aforementioned features, a big portion of demand on TEM analysis can be effectively reduced. Therefore, the STEM mode inside a modern FIB not only provides a quick means to screen the sample quality to facilitate further TEM analysis, but also a great add-on value to effectively satisfy PFA needs while minimizing unnecessary demands on TEM imaging. 
References:

[1] W. Zhao, et al., Proc. $38^{\text {th }}$ International Symposium for Testing and Failure Analysis, (2012), pp. 347 355.

[2] W. Zhao, Microscopy \& Microanalysis, Vol. 12 (Supplement 2), (2006), pp.1030 31CD.

[3] W. Zhao, Symp. Proc. the Material Research Society, 2002 Fall Meeting, (2002), Vol. 738, pp. G7.15.1 6.

[4] W. Zhao, Symp. Proc. the Material Research Society, 2002 Fall Meeting, (2002), Vol. 737, pp. F8.2.1 6.

[5] W. Zhao and D. E. Luzzi, Symp. Proc. the Material Research Society, 2000 Fall Meeting, (2000), Vol. 652, Y10.4.1 6.

[6] Thanks to GLOBALFOUNDRIES Technology Development Management and Legal teams for their supports and encouragement for the publication, and also to Mohammed Khalid Bin Dawood for his kindness in proof-reading of the manuscript.
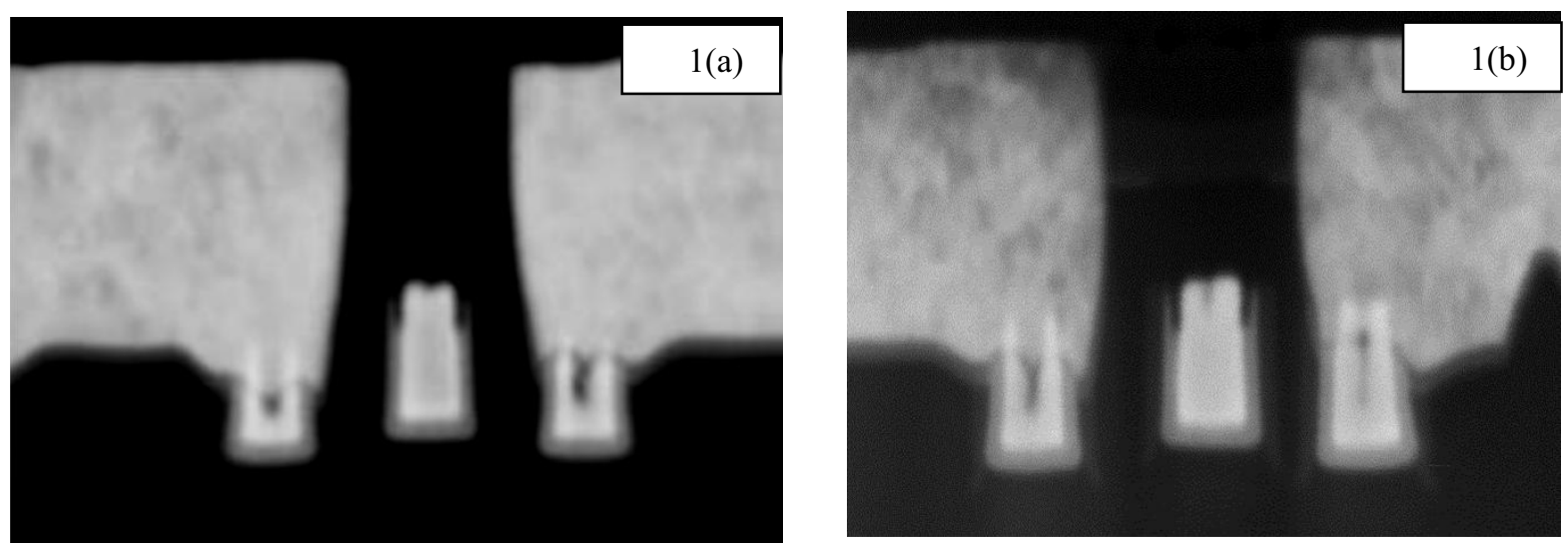

Figure 1. HAADF-STEM imaging at $30 \mathrm{kV}$ from a Helios $450 \mathrm{ML}$, for a contact in 14nm SRAM device, as displayed in (a) with a relatively thick lamella; and (b) from a thin lamella.

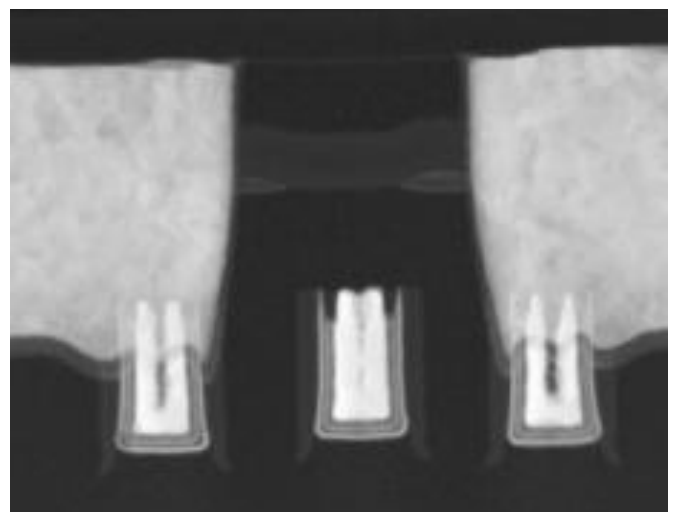

Figure 2. For a comparison, a HAADF-STEM image from a TEM with 200kV. 